\title{
Alternating Spatial Patterns for Coordinated Group Motion
}

\author{
Daniel T. Swain, Naomi Ehrich Leonard, Iain D. Couzin, Albert Kao and Rodolphe J. Sepulchre
}

\begin{abstract}
Motivated by recent observations of fish schools, we study coordinated group motion for individuals with oscillatory speed. Neighbors that have speed oscillations with common frequency, amplitude and average but different phases, move together in alternating spatial patterns, taking turns being towards the front, sides and back of the group. We propose a model and control laws to investigate the connections between these spatial dynamics, communication when sensing is range or direction limited, and convergence of coordinated group motions.
\end{abstract}

\section{INTRODUCTION}

Observations of animals that move efficiently as a group provide inspiration for design of coordinating control laws for multi-agent robotic systems. At the same time, modeling and analysis tools from systems and control theory provide possible means to better understand dynamics and robustness of natural group behaviors and how they emerge from rules at the level of individuals. Previous works include [1], [2], [3] and references therein.

In this paper we consider recent observations by Couzin and Kao of schooling fish that exhibit oscillatory acceleration and speed [4]. Preliminary data analysis suggests that the average, frequency and magnitude of the speed oscillations are similar across the group and individuals phase lock their relative speed oscillations. For example, it was observed in very small schools that neighboring fish often move together with anti-synchronized speeds; i.e., while one is speeding up, the other is slowing down. Fig. 1 shows sample data of the speeds of two fish swimming together. These observations are remarkable. They suggest possible mechanisms for information passing and collective motion in biological groups as well as new ways to synthesize coordinated motion for engineered collectives with advantageous communication and convergence properties.

For example, consider a group of individuals moving forward together at sinusoidal speed with common average, frequency and amplitude. Assume further that the speed oscillations are out of phase as in Fig. 2. Now suppose that each individual aligns itself with its neighbors by sensing and responding to the relative orientation of its neighbors. In the

D.T. Swain and N.E. Leonard are with the Mechanical and Aerospace Engineering Department, Princeton University, Princeton, NJ 08544 USA, \{dswain $\{$ naomi $\}$ aprinceton. edu

I.D. Couzin is with the Department of Ecology and Evolutionary Biology, Princeton University, Princeton, NJ 08544 USA, icouzin@princeton. edu

A. Kao is with the Physics Department, Harvard University, 17 Oxford St., Cambridge, MA 02138 USA, kao@fas. harvard. edu

R.J. Sepulchre is with the Department of Electrical Engineering and Computer Science, Université de Liège, Institut Montefiore B28, B-4000 Liège, Belgium, r.sepulchre@ulg.ac.be

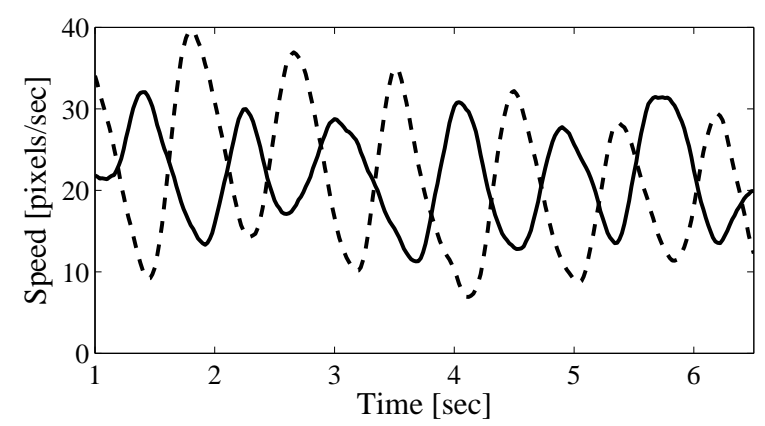

Fig. 1. Speed data for two fish swimming together in a tank. The data were produced from digitized video footage.

case that it has a blind spot behind itself, an individual can only respond to neighbors that are beside or ahead of it. If all individuals move with common speed, then individuals that start toward the back will stay in the back and have little or no influence on the group. However, with oscillatory speeds that are not synchronized, for a range of initial conditions, the individuals will alternate their positioning so that each has a chance to make relative forward progress to see and be seen at least periodically, see Fig. 2(a). In other words, the graph that describes the sensing topology among individuals may be directed under common speed conditions but becomes undirected over regular periods for out-of-phase, oscillatory speed conditions.

As a second illustration, consider a group of individuals making a turn or even moving together in a circle. Suppose the individuals start close together. If the individuals move at the same speed then individuals with larger radius of curvature will fall behind those with smaller radius of curvature. This may be a relevant behavior that adjusts relative positioning in a group. However, if the individuals move with oscillatory speed with phases that are not synchronized, then individuals can alternate their relative positioning and radius of curvature so that each is periodically in the front, on the outside, in the back and on the inside of the turning group and the group stays together, see Fig. 6. In this case too, the alternating spatial pattern enables increased communication when sensing is range or direction limited.

These illustrations reveal direct connections between the spatial dynamics, changing communication topology and convergence of group motions. Modeling and analysis of feedback control laws based on the proposed strategies can provide, for example, regions of attraction in phase space for stable coordinated group motions.

In this paper we introduce oscillatory speed into a planar 
particle model of a group of individuals. We propose and prove control laws that phase lock relative speed oscillations, building on the stabilization methodology of [5], [6]. The model is presented in Section II. In Section III we propose and prove stabilizing control laws for turning and circular patterns and explore the influence of model parameters on the resulting alternating spatial pattern. The role of oscillatory speed in the case of limited communication is explored in Section IV.

\section{PARTICLE MODEL}

We consider a group of $N$ individuals and model each as a particle with unit mass. In this paper we restrict motion to the plane and identify $\mathbb{R}^{2}$ with $\mathbb{C}$. For $k=1, \ldots, N$, let $r_{k} \in \mathbb{C}$ denote the position of particle $k$ and $f_{k} \in \mathbb{C}$ the total external force on particle $k$. Let $\alpha_{k}=\left|\dot{r}_{k}\right|$ be the speed of particle $k$ and for $\alpha_{k} \neq 0$, let $\theta_{k} \in S^{1}$ be the direction of motion of particle $k$ relative to an inertial frame. We can express the velocity of particle $k$ as

$$
\dot{r}_{k}=\alpha_{k} e^{i \theta_{k}}
$$

and the dynamics of particle $k$ as

$$
\ddot{r}_{k}=\left(\dot{\alpha}_{k}+\alpha_{k} \dot{\theta}_{k} i\right) e^{i \theta_{k}}
$$

for $k=1, \ldots, N$. The first term on the right of (1) is the component of force in the direction of motion and the second term is the component of force normal to velocity. We decompose the control into two corresponding components:

$$
\begin{aligned}
& \dot{\theta}_{k}=u_{k} \\
& \dot{\alpha}_{k}=v_{k} .
\end{aligned}
$$

\section{A. Constant Speed}

Several authors have studied stabilization of collective motion in the case that $v_{k}=0$ and $\alpha_{k}=\alpha$ for $k=1, \ldots, N$; see for example [7], [5]. In this case, where constant unit speed for each particle is assumed, the dynamics simplify to

$$
\begin{aligned}
& \dot{r}_{k}=e^{i \theta_{k}} \\
& \dot{\theta}_{k}=u_{k} .
\end{aligned}
$$

If $u_{k}$ depends only on $\boldsymbol{\theta}=\left(\theta_{1}, \ldots, \theta_{N}\right)$ and not on $\mathbf{r}=$ $\left(r_{1}, \ldots, r_{N}\right)$, then the oscillator dynamics (3) are decoupled.

Following [8], [9] the complex order parameter for a set of phase angles $\boldsymbol{\psi}=\left\{\psi_{1}, \ldots, \psi_{N}\right\} \in T^{N}$, is defined as

$$
p_{\psi}=\frac{1}{N} \sum_{k=1}^{N} e^{i \psi_{k}} \text {. }
$$

Its magnitude $\left|p_{\psi}\right|$ gives a measure of synchronization. When $\left|p_{\psi}\right|=1$ the phases are synchronized, i.e., $\psi_{k}=\psi_{j}$ for all $j, k$. When $p_{\psi}=0$ the phases are balanced. The phases are in a symmetric balanced configuration called the splay state when they are evenly distributed around the unit circle.

The gradient of the phase potential

$$
U_{1}(\boldsymbol{\psi})=\frac{N}{2}\left|p_{\psi}\right|^{2}
$$

yields the coupled oscillator dynamics

$$
\begin{aligned}
\dot{\psi}_{k} & =\zeta-K \frac{\partial U_{1}}{\partial \psi_{k}}=\zeta-K\left\langle p_{\psi}, i e^{i \psi_{k}}\right\rangle \\
& =\zeta-\frac{K}{N} \sum_{j=1}^{N} \sin \psi_{j k},
\end{aligned}
$$

where $\psi_{j k}=\psi_{j}-\psi_{k}$ and the inner product is defined by $\left\langle\boldsymbol{z}_{1}, \boldsymbol{z}_{2}\right\rangle=\operatorname{Re}\left\{\overline{\boldsymbol{z}}_{1}^{T} \boldsymbol{z}_{2}\right\}$ for $\boldsymbol{z}_{1}, \boldsymbol{z}_{2} \in \mathbb{C}^{N}$. This is the Kuramoto model in the case of identical natural frequencies $\zeta$ [8], [9]. When $K>0$, the set of balanced solutions is stabilized. When $K<0$, the synchronized state is stabilized.

In [5] the authors consider both the phase and spatial dynamics of the particle model and design control laws $u_{k}=u_{k}(\boldsymbol{\theta}, \mathbf{r})$ to stabilize coordinated patterns in relative phase and relative position for the particle model (2)-(3). The control laws derive from a superposition of phase potentials and spacing potentials for stabilization of parallel and circular motions of the particle group. In the case of circular motion, the particles are stabilized to move around the same circle with either synchronized or balanced phasing. A control law is derived from potentials dependent on integer multiples of phase angles and stabilizes circular motion of particles with phasing in symmetric patterns. For example, the phases can be stabilized to the splay state so that the particles are uniformly distributed as they rotate around a circle.

In [6], the authors generalize the stabilization of collective motion to a particle group with limited communication. A graph and the corresponding Laplacian matrix represent the communication topology. The phase and spacing potentials are generalized by defining them in terms of a Laplaciandependent quadratic form. In the case of fixed topology (constant Laplacian) and undirected graph (every communication link is bi-directional), stabilization follows under certain connectedness conditions. In the case of time-varying and directed graphs, stabilization is proven only with the addition of consensus dynamics and the communicating of the consensus variables.

\section{B. Oscillating Speed}

We introduce to the particle model a sinusoidal variation about a common nominal speed, taken here to be unit speed. We assume that there is a feedback loop on each control input $v_{k}$ so that particle speed is controlled tightly to the desired speed; accordingly, we design $\alpha_{k}(t)$ instead of $v_{k}$. We introduce a new input $g_{k}$ which controls the phase of the speed oscillation. Consider the speed profile for $k=$ $1, \ldots, N$

$$
\alpha_{k}(t)=1+\mu \cos \phi_{k}(t)
$$

where constant $\mu \in(0,1)$ is the amplitude of the oscillations and $\phi_{k}(t)$ is an instantaneous phase angle. The particle model becomes

$$
\begin{aligned}
\dot{r}_{k} & =\left(1+\mu \cos \phi_{k}(t)\right) e^{i \theta_{k}(t)} \\
\dot{\theta}_{k} & =u_{k} \\
\dot{\phi}_{k} & =g_{k} .
\end{aligned}
$$


We are interested in choices of $g_{k}$ such that at steadystate $\dot{\phi}_{k}=\Omega$ where $\Omega \in \mathbb{R}$ is a constant and $\alpha_{k}(t)=$ $1+\mu \cos \left(\Omega t+\phi_{0}\right)$ for some $\phi_{0} \in S^{1}$.

For the headings $\boldsymbol{\theta}=\left\{\theta_{1}, \ldots, \theta_{N}\right\}$ and speed phases $\phi=\left\{\phi_{1}, \ldots, \phi_{N}\right\}$, we define synchronization parameters $p_{\theta}$ and $p_{\phi}$ and potentials $U_{1}(\boldsymbol{\theta})$ and $U_{1}(\boldsymbol{\phi})$ as per (4) and (5), respectively. Gradient dynamics corresponding to these potentials are

$$
\begin{aligned}
& \dot{\theta}_{k}=u_{k}=\omega-K_{\theta} \frac{\partial U_{1}(\boldsymbol{\theta})}{\partial \theta_{k}} \\
& \dot{\phi}_{k}=g_{k}=\Omega-K_{\phi} \frac{\partial U_{1}(\boldsymbol{\phi})}{\partial \phi_{k}}
\end{aligned}
$$

with scalar constants $\omega, K_{\theta}$, and $K_{\phi}$. Here $u_{k}$ depends only on relative headings $\theta_{j k}$ and $g_{k}$ depends only on relative speed phases $\phi_{j k}$. The stability results are straightforward; for example, if $K_{\theta}>0$ and $K_{\phi}<0$, the headings synchronize and the speed phases balance.

Fig. 2 shows simulation results for the particle model (8) with common constant heading $\theta_{k}=\theta_{0}, \dot{\theta}_{k}=0$ and sinusoidal oscillations $\dot{\phi}_{k}=\Omega$ for $k=1,2,3$. The initial speed phase angles for the three particles are chosen to be out of phase. The particles move in straight parallel lines and the relative position of the particles alternates as a result of the out-of-phase locked speed oscillations.

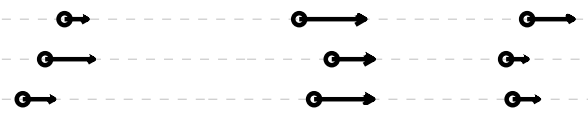

(a)

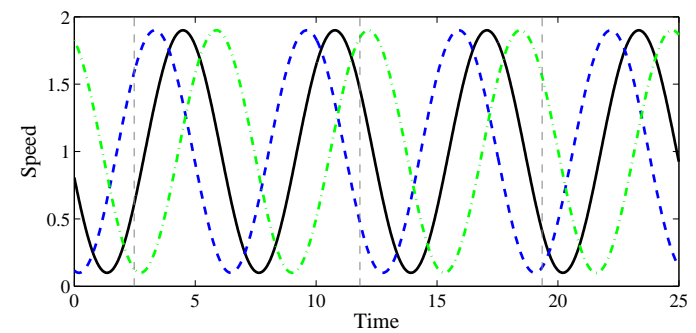

(b)

Fig. 2. Particle model simulation results with speed oscillations and constant heading. (a) Particle locations and velocities at three time instants corresponding to the three vertical dashed lines in (b) the speed of each particle as a function time.

\section{CIRCULAR PATTERNS WITH SPEED OSCILLATION}

In this section we design control laws to stabilize circular motion with alternating positioning of the particle group for the particle dynamics (8). We first examine steady-state solutions and the influence of the parameters $\mu, \omega, \Omega$. The stabilization results follow.

\section{A. Steady-State, Alternating, Circular Motion Patterns}

Solutions of (8) under the steady-state conditions $\dot{\theta}_{k}=$ $\omega$ and $\dot{\phi}_{k}=\Omega, k=1, \ldots, N$, are described by the decomposition

$$
r_{k}=c_{k}+R\left(\theta_{k}\right)+\mu e^{i \theta_{k}} E\left(\phi_{k}\right)
$$

where

$$
R\left(\theta_{k}\right)=-i \omega^{-1} e^{i \theta_{k}}
$$

defines a circle of radius $\left|\omega^{-1}\right|$ and

$$
E\left(\phi_{k}\right)=\frac{1}{\Omega^{2}-\omega^{2}}\left(\Omega \sin \phi_{k}+i \omega \cos \phi_{k}\right)
$$

defines an ellipse with eccentricity $\frac{\Omega}{\omega}$ and scale $\left|\Omega^{2}-\omega^{2}\right|^{-1}$. As illustrated in Fig. 3, the steady-state trajectory of particle $k$ corresponds to motion around an ellipse that rotates around a circle of radius $\left|\omega^{-1}\right|$ centered at $c_{k}$. When $\omega \neq \Omega$ the steady-state orbit remains inside an annulus defined by

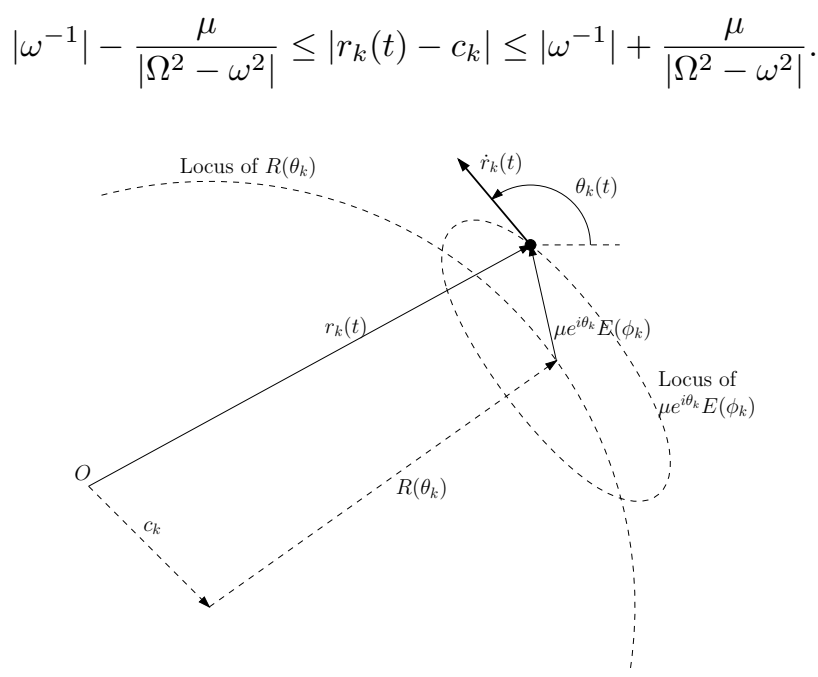

Fig. 3. Decomposition of $r_{k}(t)$ into circular and elliptical components.

We now consider the influence of relative values of $\omega, \Omega$, and $\mu$ on the circular motion pattern. If $\mu=0$, we recover the case with no speed oscillations and obtain closed orbits on circles of radius $\left|\omega^{-1}\right|$ as in Fig. 4(a). In the case that $\omega=\Omega$, there is a singularity (see (10)). This manifests as a constant velocity term and hence spiral trajectories that do not remain bounded. This is illustrated in Fig. 4(b).

For $\Omega>\omega$, the $\mu E\left(\phi_{k}\right)$ ellipse has its semi-major axis tangent to the $R\left(\theta_{k}\right)$ circle for each value of $\theta_{k}$ and the orbits have the appearance of rounded-out polygons as in Figs. 4(c) and 4(d). In fact, if $\frac{\Omega}{\omega}$ is an integer, the orbits approximate a polygon with $\frac{\Omega}{\omega}$ sides (Fig. 4(c)).

For $\Omega<\omega$, the $\mu E\left(\phi_{k}\right)$ ellipse has its semi-major axis parallel to the radius vector of the $R\left(\theta_{k}\right)$ circle for each value of $\theta_{k}$ and the orbits have a cardioid appearance as in Figs. 4(e) and 4(f). In this case the ratio $\frac{\Omega}{\omega}$ determines the number of loops the orbit completes as $\theta_{k}$ goes through a $2 \pi$ cycle.

We also observe that the orbit is closed and periodic whenever $\frac{\Omega}{\omega} \neq 1$ is rational (Figs. 4(c) and 4(e)), and that the orbits are aperiodic otherwise (Figs. 4(d) and 4(f)).

\section{B. Stabilization of Circular Motion Patterns}

We now turn to the stabilization of a collection of $N$ particles moving according to the dynamics (8). Our goal is to find control laws for the steering control $u_{k}$ and speed phase control $g_{k}$ that stabilize the particle trajectories 


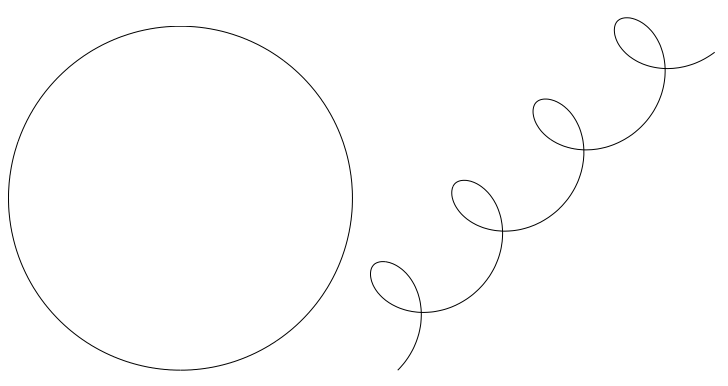

(a)

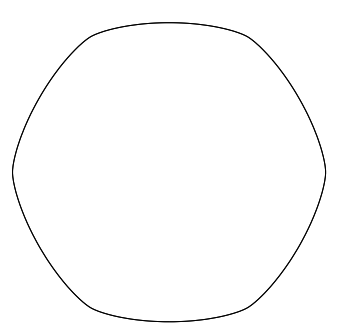

(c)

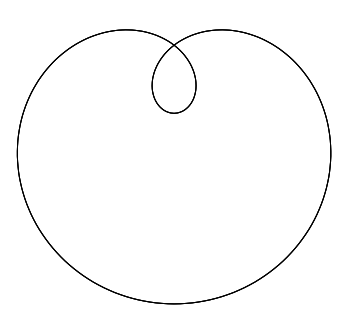

(e) (b)

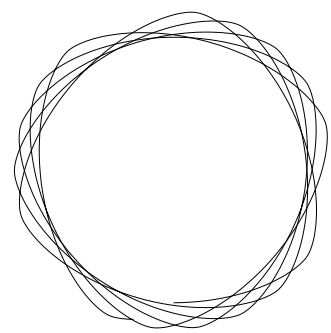

(d)

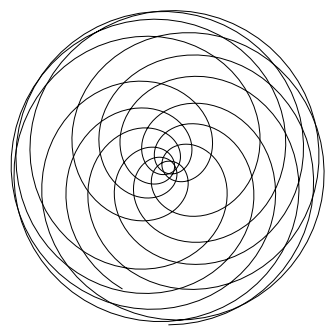

(f)

Fig. 4. Steady state solutions of (8) for an arbitrary particle. (a) Circular orbit when $\mu=0$; (b) Spiral trajectory for $\Omega=\omega$; (c) Closed hexagonal orbit when $\Omega=6 \omega$; (d) Non-closed orbit when $\Omega=\pi \omega$; (e) Closed cardioid orbit when $2 \Omega=\omega$; (f) Non-closed orbit when $e \Omega=\omega$.

to steady-state orbits such as those described by (9) with $\dot{\theta}_{k}=\omega \neq 0, \dot{\phi}_{k}=\Omega$ and $c_{k}=c_{0}$ for some $c_{0} \in \mathbb{C}$ and $k=1, \ldots, N$. In our first result we address this goal without imposing prescribed phase locking patterns on the relative headings $\theta_{j k}$ and relative speed phases $\phi_{j k}$.

Inspired by [5], we define

$$
s_{k}=-i \omega c_{k}=e^{i \theta_{k}}-i \omega r_{k}+i \omega \mu e^{i \theta_{k}} E\left(\phi_{k}\right)
$$

and the corresponding vector $s=\left\{s_{1}, \ldots, s_{n}\right\} \in \mathbb{C}^{N}$. The algebraic condition

$$
P s=0, P=I_{N}-\frac{1}{N} \mathbf{1 1}^{T}
$$

corresponds to the desired condition that $c_{k}=c_{0}$ for some $c_{0}$ and for all $k=1, \ldots, N$. This condition, along with $\dot{\theta}_{k}=\omega$ and $\dot{\phi}_{k}=\Omega$, describes our desired steady-state.

Theorem 1: For the speed-oscillating particle model (8) with steering control and phase controls

$$
\begin{aligned}
u_{k} & =\omega-\kappa\left\langle P_{k} s, i e^{i \theta_{k}}\right\rangle, \kappa \in \mathbb{R}^{+} \\
g_{k} & =\frac{\Omega}{\omega} u_{k}
\end{aligned}
$$

respectively, all solutions converge to patterns defined by motion about a circle of common center and radius $\left|\omega^{-1}\right|$ with superimposed motion due to the speed oscillations as defined by (9).

Proof: Following the strategy of [5], we choose a candidate Lyapunov function

$$
S(\boldsymbol{r}, \boldsymbol{\theta}, \boldsymbol{\phi})=\frac{1}{2}\|P \boldsymbol{s}\|^{2}
$$

(recall that $\boldsymbol{r}, \boldsymbol{\theta}$, and $\boldsymbol{\phi}$ are vectors of the $r_{k}, \theta_{k}$, and $\phi_{k}$ variables, respectively), which has time-derivative

$$
\dot{S}=\langle P s, P \dot{s}\rangle=\sum_{k=1}^{N}\left\langle P_{k} s, \dot{s}_{k}\right\rangle,
$$

where $P_{k}$ is the $k^{\text {th }}$ row of $P$ and we have taken advantage of the fact that $P^{2}=P$ is a projector.

With controls (13) and (14), it follows that along solutions of (8) we have

$$
\dot{S}=-\sum_{k=1}^{N}\left(1+\mu \cos \phi_{k}\right) \kappa\left\langle P_{k} s, i e^{i \theta_{k}}\right\rangle^{2} \leq 0 .
$$

Since (17) is nonincreasing, solutions converge to the largest invariant set $\Lambda$ on $\dot{S}=0$, corresponding to the condition

$$
\left\langle P_{k} \boldsymbol{s}, i e^{i \theta_{k}}\right\rangle=0
$$

for each $k=1, \ldots, N$. Plugging (18) into (13) gives $\dot{\theta}_{k}=$ $u_{k}=\omega$ for all $k$. By (14) this implies that $\dot{\phi}_{k}=g_{k}=\Omega$ for all $k$. Also, since $\dot{\theta}_{k}=\omega \neq 0$ for all $k,(18)$ is only satisfied if $P s=0$. Together these conditions imply convergence to the desired steady-state.

Remark. By (11) and the form of (6), the control law (13) can be rewritten as

$$
u_{k}=\kappa \frac{\partial U_{1}(\boldsymbol{\theta})}{\partial \theta_{k}}+\omega\left(1+\kappa\left\langle\tilde{r}_{k}, e^{i \theta_{k}}\right\rangle\right)+\mu \kappa \omega\left\langle\tilde{e}_{k}, e^{i \theta_{k}}\right\rangle
$$

where

$$
\tilde{r}_{k}=P_{k} \boldsymbol{r}=r_{k}-\frac{1}{N} \sum_{j=1}^{N} r_{j}
$$

is the relative position of $r_{k}$ from the group center of mass and

$$
\tilde{e}_{k}=P_{k} \boldsymbol{e}=e^{i \theta_{k}} E\left(\phi_{k}\right)-\frac{1}{N} \sum_{j=1}^{N} e^{i \theta_{j}} E\left(\phi_{j}\right),
$$

where $e=\left\{e^{i \theta_{1}} E\left(\phi_{1}\right), \ldots, e^{i \theta_{N}} E\left(\phi_{N}\right)\right\}$. Furthermore,

$$
\left\langle\tilde{e}_{k}, e^{i \theta_{k}}\right\rangle=\operatorname{Re}\left\{E\left(\phi_{k}\right)\right\}-\frac{1}{N} \sum_{j=1}^{N}\left\langle E\left(\phi_{j}\right), e^{i \theta_{k j}}\right\rangle,
$$

in which the term $\operatorname{Re}\left\{E\left(\phi_{k}\right)\right\}$ is proportional to the component of acceleration in the particle $k$ direction of motion. Similarly, $\frac{1}{N} \sum_{j=1}^{N}\left\langle E\left(\phi_{j}\right), e^{i \theta_{k j}}\right\rangle$ can be interpreted as being proportional to the average component of the group's acceleration (assuming that it is in steady-state) projected also into the particle $k$ direction of motion. The term $\frac{\partial U_{1}(\boldsymbol{\theta})}{\partial \theta_{k}}$ is a function only of relative headings, see (6). Hence, the 
control law (19) can be computed from relative physical quantities. When $\mu=0$, this control is identical to that produced in [5].

Fig. 5 shows results of simulations in which (13) is used to stabilize collective motion of patterns similar to those in Fig. 4. In this case, we set $e \Omega=\omega$ and particles converge to patterns similar to Fig. 4(f), where the trajectories share a common center. We note that Fig. 5 bears a striking resemblance to the "random" circulation of a fish shoal about a common center [10]. Indeed the steady-state motion reflects a behavior in which individuals cycle between periods of higher velocity (when $\cos \phi_{k}$ is near +1 ) and periods of lower velocity (when $\cos \phi_{k}$ is near -1 ). In maintaining a constant angular frequency $\omega$, this behavior in turn produces cycles between particle motion close to the outside perimeter of the annulus (large $\left|r_{k}-c_{0}\right|$ ) and particle motion near the inside perimeter of the annulus (small $\left|r_{k}-c_{0}\right|$ ).

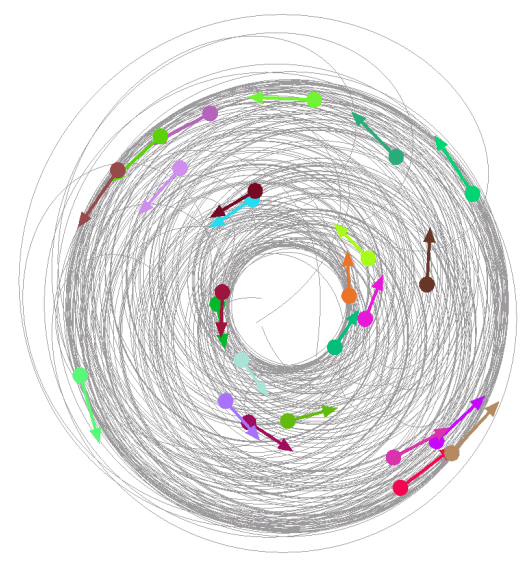

Fig. 5. Simulation results using the steering control (13) with speed phasing control (14). Solid circles show positions, arrow directions indicate heading and arrow lengths indicate speed. $N=25, \mu=0.5, e \Omega=\omega$.

\section{Stabilization of Circular Motion Patterns with Prescribed Relative Phases}

To stabilize circular motion patterns with prescribed relative heading angles and relative speed phases we augment the steering control (13) and speed phasing control (14) with gradients of potentials of the form derived in [5]. Our goal is to stabilize the group so that it moves as a cluster around a circle with alternating positioning of the particles in the cluster. To cluster the group we synchronize the headings $\theta_{k}$. To enforce alternating positions we balance the speed phases $\phi_{k}$. As a particular example here we stabilize the speed phases $\phi_{k}$ to the splay state. The modified control laws are

$$
\begin{aligned}
u_{k}= & \omega\left(1+\kappa\left\langle\tilde{r}_{k}, e^{i \theta_{k}}\right\rangle\right)+\mu \kappa \omega\left\langle\tilde{e}_{k}, e^{i \theta_{k}}\right\rangle \\
& -\frac{\partial\left(U_{\theta}(\boldsymbol{\theta})-\kappa U_{1}(\boldsymbol{\theta})\right)}{\partial \theta_{k}} \\
g_{k}= & \frac{\Omega}{\omega} u_{k}-\frac{\partial\left(U_{\phi}(\boldsymbol{\phi})-U_{1}(\boldsymbol{\phi})\right)}{\partial \phi_{k}},
\end{aligned}
$$

where $U_{\theta}(\boldsymbol{\theta})=K_{\theta} U_{1}(\boldsymbol{\theta})$ synchronizes the heading angles $\theta_{k}$ for $K_{\theta}<0$ and

$$
U_{\phi}(\phi)=\sum_{m=1}^{\left\lfloor\frac{N}{2}\right\rfloor} K_{\phi, m}\left|\frac{1}{m N} \sum_{j=1}^{N} e^{i m \theta_{j}}\right|^{2},
$$

where $\left\lfloor\frac{N}{2}\right\rfloor$ is the largest integer smaller than or equal to $\frac{N}{2}$, stabilizes the splay state of $\phi$ when $K_{\phi, m}>0, m=$ $1, \ldots,\left\lfloor\frac{N}{2}\right\rfloor$. Proof of stabilization for these controlled dynamics will appear in a forthcoming paper.

Fig. 6 shows simulation results when $\Omega=4 \omega, K_{\theta}<0$, and $K_{\phi, m}>0, m=1, \ldots,\left\lfloor\frac{N}{2}\right\rfloor$. Since $K_{\theta}<0$, the heading angles $\theta_{k}$ synchronize and all of the individual particles converge to the same ellipse which moves around the same circle. Additionally, since $K_{\phi, m}>0$, the speed phases stabilize to the splay state and the particles are evenly distributed around the ellipse defined by $\mu E(\phi)$. The shape and size of the ellipse are controlled by parameters $\mu, \omega$, and $\Omega$.

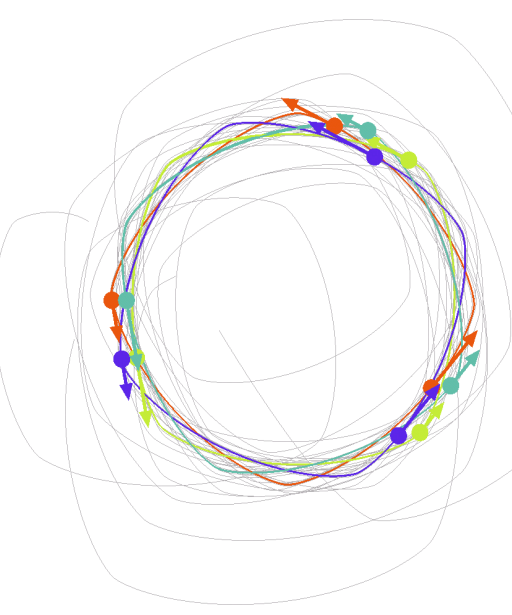

Fig. 6. Simulation results using the steering control (20) with speed phase control (21). Solid circles show positions, arrow directions indicate heading and arrow lengths indicate speed. $N=4, \mu=0.9, \Omega=4 \omega$. Three snapshots of the same cluster are shown.

\section{SPEED PHASE STABILIZATION WITH LIMITED COMMUNICATION}

In the case that individuals in the group have limited communication that is time-invariant and undirected, we can use the Laplacian-dependent spacing and phase potentials of [6] to generalize the stabilization results of Section III. Here we are interested in the advantages of alternating spatial patterns when communication is range or direction limited as motivated in the introduction (e.g., fish with rear blind spots). In this case the communication is time-varying and directed, but the alternating positioning makes improved communication over regular time intervals possible.

To make a preliminary investigation we focus only on the coupled speed phase dynamics. To do this we fix the rest of the dynamics so that the particles move on the steady-state pattern and allow only the speed phases $\phi_{k}$ to be controlled 
with feedback and limited communication. Accordingly, we consider the modified model

$$
\begin{aligned}
\dot{r}_{k} & =\left(1+\mu \cos \phi_{k}\right) e^{i \theta_{k}}+\mu \bar{g}_{k} e^{i \theta_{k}} \frac{\partial E}{\partial \phi_{k}} \\
\dot{\theta}_{k} & =u_{k}=\omega \\
\dot{\phi}_{k} & =g_{k}=\Omega+\bar{g}_{k}
\end{aligned}
$$

with initial conditions chosen so that $r_{k}(0)=R\left(\theta_{k}(0)\right)+$ $\mu e^{i \theta_{k}(0)} E\left(\phi_{k}(0)\right)$ and $\theta_{k}(0)=\theta_{j}(0)$ for all $k, j$. This corresponds to all particles moving about the same ellipse which rotates around a circle as in the simulation example of Fig. 6. The additional $\mu \bar{g}_{k} e^{i \theta_{k}} \frac{\partial E}{\partial \phi_{k}}$ term in (23) keeps particles on the ellipse for arbitrary control law $g_{k}=\Omega+\bar{g}_{k}$, so that control law $\bar{g}_{k}$ can be designed to stabilize prescribed relative speed phases.

Let each particle be represented by a node on a graph $G(t)$ with edge set $\mathcal{E}(t)$ defining communication links such that $(k, j) \in \mathcal{E}(t)$ if particle $k$ can sense particle $j$ at time $t$. The neighbor set $\mathcal{N}_{k}(t)$ of particle $k$ contains all $j$ such that $(k, j) \in \mathcal{E}(t)$ at time $t$ and $\left|\mathcal{N}_{k}(t)\right|$ is its cardinality. We also require a notion of graph connectedness over time periods. A graph is said to be uniformly connected if there exists a node $i$ and a time $T>0$ such that for all $t$ node $i$ is connected to all other nodes across the intervals $[t, t+T]$ (see [6]).

Theorem 2: Consider the model (23) with the initial conditions specified above and sensing for each particle limited by a rear blindspot angle $\beta<\pi$ as shown in Fig. 7. The speed phase control

$$
g_{k}=\Omega+\frac{K_{\phi}}{\left|\mathcal{N}_{k}(t)\right|} \sum_{j \in \mathcal{N}_{k}(t)} \sin \phi_{j k}
$$

for some $K_{\phi}<0$ and $\left|K_{\phi}\right|<|\Omega|$, exponentially stabilizes the synchronized state of $\phi_{k}=\phi_{j}$, for all $k, j$ and $\dot{\phi_{k}}=\Omega$.

Proof: The control (24) specifies Laplacian consensus dynamics on $\phi \in T^{N}$. By [11] the synchronized state is exponentially stable when $K_{\phi}<0$ if the communication graph is uniformly connected.

Since $\left|K_{\phi}\right|<|\Omega|$, we have $\left|\dot{\phi}_{k}\right| \geq|\Omega|-\left|K_{\phi}\right|>0$ for all $t$. Hence, each particle will make a complete cycle around the $E\left(\phi_{k}\right)$ ellipse at least once every $T=\frac{2 \pi}{|\Omega|-\left|K_{\phi}\right|}$ time units. By (23), the $E\left(\phi_{k}\right)$ are constrained to be identical and when any particle is at the rear it can sense every other particle on the ellipse. Therefore, the graph is uniformly connected for any node $i$ and some $T \in\left[\frac{2 \pi}{|\Omega|+\left|K_{\phi}\right|}, \frac{2 \pi}{|\Omega|-\left|K_{\phi}\right|}\right]$ and the $\phi$ converge to a synchronized steady-state with $\dot{\phi}_{k}=\Omega$.

Theorem 2 yields synchronization of speed phases, however it may be more desirable to balance them or even stabilize them to the splay state so that particles do not collide but rather distribute themselves uniformly within their cluster. Following [12] and [6] we can stabilize balanced patterns such as the splay state for a directed, time-varying graph by using a dynamic consensus algorithm in which a consensus variable is shared amongst communicating individuals. Simulation results for this case are shown in Fig. 7.

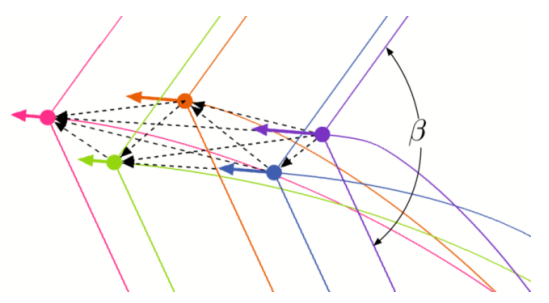

Fig. 7. Simulation of a formation with limited communication defined by a rear blind spot angle of $\beta=2 \pi / 3$. The oscillating speed phases converge to a balanced phase arrangement, as prescribed. Dashed lines connecting particles represent sensing links. Note that the particle in the rear can sense all of the other particles.

\section{CONCLUSION}

Motivated by observations of fish schooling, we study collective motion of individuals with oscillatory speed. The relative positions of individuals in a group will cycle when individual speeds are oscillatory and out-of-phase locked. This leads to alternating spatial patterns which yields a rich family of group motions and increased communication when sensing is distance or direction limited. In particular, each individual will alternately be at the front of the group where it can be sensed by others and in the rear of the group where it can sense others. In the case of turning motion, each individual will also cycle between the inside and the outside of the turn (see Fig. 5). The results in this paper provide a new avenue for connecting group dynamics with changing communication topology and convergence of collective motion patterns.

\section{REFERENCES}

[1] A. Jadbabaie, J. Lin, and A. S. Morse, "Coordination of groups of mobile autonomous agents using nearest neighbor rules," IEEE Trans. Automatic Control, vol. 48, pp. 988-1001, 2003.

[2] R. Olfati-Saber, "Flocking for multi-agent dynamic systems: Algorithms and theory," IEEE Trans. on Automatic Control, vol. 51, pp. 401-421, 2006.

[3] B. W. Andrews, K. M. Passino, and T. A. Waite, "Social foraging theory for robust multiagent system design," IEEE Transactions on Automation Science and Engineering, vol. 4, pp. 79-86, 2007.

[4] I. D. Couzin and A. Kao, "Fish schooling." Preprint, 2006.

[5] R. Sepulchre, D. Paley, and N. Leonard, "Stabilization of planar collective motion: All-to-all communication," IEEE Transactions on Automatic Control, vol. 52, pp. 811-824, 2007.

[6] R. Sepulchre, D. Paley, and N. Leonard, "Stabilization of planar collective motion with limited communication," IEEE Transactions on Automatic Control. To appear.

[7] E. Justh and P. Krishnaprasad, "Equilibria and steering laws for planar formations," Systems and Control Letters, vol. 52, no. 1, pp. 25-38, 2004.

[8] Y. Kuramoto, Chemical oscillations, waves, and turbulence. SpringerVerlag, 1984.

[9] S. H. Strogatz, "From Kuramoto to Crawford: exploring the onset of synchronization in populations of coupled oscillators," Physica D, vol. 143 , pp. 1-20, 2000.

[10] I. Couzin, J. Krause, R. James, G. Ruxton, and N. Franks, "Collective memory and spatial sorting in animal groups," J. Theor. Biology, no. 218, pp. 1-11, 2002.

[11] L. Moreau, "Stability of multiagent systems with time-dependent communication links," IEEE Trans. Automatic Control, vol. 50, no. 2, pp. 169-182, 2005.

[12] L. Scardovi, A. Sarlette, and R. Sepulchre, "Synchronization and balancing on the $N$-torus," Systems and Control Letters, vol. 56, pp. 335-341, 2007. 